\title{
Research Article \\ General Iterative Algorithms for Hierarchical Fixed Points Approach to Variational Inequalities
}

\author{
Nopparat Wairojjana and Poom Kumam \\ Department of Mathematics, Faculty of Science, King Mongkut's University of Technology Thonburi \\ (KMUTT), Bangmod, Bangkok 10140, Thailand \\ Correspondence should be addressed to Poom Kumam, poom.kum@kmutt.ac.th
}

Received 24 March 2012; Accepted 16 May 2012

Academic Editor: Zhenyu Huang

Copyright (C 2012 N. Wairojjana and P. Kumam. This is an open access article distributed under the Creative Commons Attribution License, which permits unrestricted use, distribution, and reproduction in any medium, provided the original work is properly cited.

This paper deals with new methods for approximating a solution to the fixed point problem; find $\tilde{x} \in F(T)$, where $H$ is a Hilbert space, $C$ is a closed convex subset of $H, f$ is a $\rho$-contraction from $C$ into $H, 0<\rho<1, A$ is a strongly positive linear-bounded operator with coefficient $\bar{\gamma}>0$, $0<\gamma<\bar{\gamma} / \rho, T$ is a nonexpansive mapping on $C$, and $P_{F(T)}$ denotes the metric projection on the set of fixed point of $T$. Under a suitable different parameter, we obtain strong convergence theorems by using the projection method which solves the variational inequality $\langle(A-\gamma f) \tilde{x}+\tau(I-S) \tilde{x}, x-\tilde{x}\rangle \geq 0$ for $x \in F(T)$, where $\tau \in[0, \infty)$. Our results generalize and improve the corresponding results of Yao et al. (2010) and some authors. Furthermore, we give an example which supports our main theorem in the last part.

\section{Introduction}

Throughout this paper, we assume that $H$ is a real Hilbert space where inner product and norm are denoted by $\langle\cdot, \cdot\rangle$ and $\|\cdot\|$, respectively, and let $C$ be a nonempty closed convex subset of $H$. A mapping $T: C \rightarrow C$ is called nonexpansive if

$$
\|T x-T y\| \leq\|x-y\|, \quad \forall x, y \in C .
$$

We use $F(T)$ to denote the set of fixed points of $T$, that is, $F(T)=\{x \in C: T x=x\}$. It is assumed throughout the paper that $T$ is a nonexpansive mapping such that $F(T) \neq \emptyset$.

Recall that a mapping $f: C \rightarrow H$ is a contraction on $C$ if there exists a constant $\rho \in(0,1)$ such that

$$
\|f(x)-f(y)\| \leq \rho\|x-y\|, \quad \forall x, y \in C .
$$


A mapping $A: H \rightarrow H$ is called a strongly positive linear bounded operator on $H$ if there exists a constant $\bar{\gamma}>0$ with property

$$
\langle A x, x\rangle \geq \bar{\gamma}\|x\|^{2}, \quad \forall x \in H
$$

A mapping $M: H \rightarrow H$ is called a strongly monotone operator with $\alpha$ if

$$
\langle x-y, M x-M y\rangle \geq \alpha\|x-y\|^{2}, \quad \forall x, y \in H,
$$

and $M$ is called a monotone operator if

$$
\langle x-y, M x-M y\rangle \geq 0, \quad \forall x, y \in H
$$

We easily prove that the mapping $(I-T)$ is monotone operator, if $T$ is nonexpansive mapping.

The metric (or nearest point) projection from $H$ onto $C$ is mapping $P_{C}[\cdot]: H \rightarrow C$ which assigns to each point $x \in C$ the unique point $P_{C}[x] \in C$ satisfying the property

$$
\left\|x-P_{C}[x]\right\|=\inf _{y \in C}\|x-y\|=: d(x, C)
$$

The variational inequality for a monotone operator, $M: H \rightarrow H$ over $C$, is to find a point in

$$
\mathrm{VI}(C, M):=\{\tilde{x} \in C:\langle x-\tilde{x}, M \tilde{x}\rangle \geq 0, \forall x \in C\}
$$

A hierarchical fixed point problem is equivalent to the variational inequality for a monotone operator over the fixed point set. Moreover, to find a hierarchically fixed point of a nonexpansive mapping $T$ with respect to another nonexpansive mapping $S$, namely, we find $\tilde{x} \in F(T)$ such that

$$
\langle x-\tilde{x},(I-S) \tilde{x}\rangle \geq 0, \quad \forall x \in F(T) .
$$

Iterative methods for nonexpansive mappings have recently been applied to solve a convex minimization problem; see, for example, [1-5] and the references therein. A typical problem is to minimize a quadratic function over the set of the fixed points of a nonexpansive mapping on a real Hilbert space $H$ :

$$
\min _{x \in F(T)} \frac{1}{2}\langle A x, x\rangle-\langle x, b\rangle
$$

where $b$ is a given point in $H$. In [5], it is proved that the sequence $\left\{x_{n}\right\}$ defined by the iterative method below, with the initial guess $x_{0} \in H$ chosen arbitrarily,

$$
x_{n+1}=\left(I-\alpha_{n} A\right) T x_{n}+\alpha_{n} b, \quad n \geq 0,
$$


converges strongly to the unique solution of the minimization problem (1.9) provided the sequence $\left\{\alpha_{n}\right\}$ of parameters satisfies certain appropriate conditions.

On the other hand, Moudafi [6] introduced the viscosity approximation method for nonexpansive mappings (see [7] for further developments in both Hilbert and Banach spaces). Starting with an arbitrary initial $x_{0} \in H$, define a sequence $\left\{x_{n}\right\}$ recursively by

$$
x_{n+1}=\sigma_{n} f\left(x_{n}\right)+\left(1-\sigma_{n}\right) T x_{n}, \quad n \geq 0,
$$

where $\left\{\sigma_{n}\right\}$ is a sequence in $(0,1)$. It is proved in $[6,7]$ that under certain appropriate conditions imposed on $\left\{\sigma_{n}\right\}$, the sequence $\left\{x_{n}\right\}$ generated by (1.11) strongly converges to the unique solution $x^{*}$ in $C$ of the variational inequality

$$
\left\langle(I-f) x^{*}, x-x^{*}\right\rangle \geq 0, \quad x \in C
$$

In 2006, Marino and Xu [8] introduced a general iterative method for nonexpansive mapping. Starting with an arbitrary initial $x_{0} \in H$, define a sequence $\left\{x_{n}\right\}$ recursively by

$$
x_{n+1}=\epsilon_{n} \gamma f\left(x_{n}\right)+\left(I-\epsilon_{n} A\right) T x_{n}, \quad n \geq 0 .
$$

They proved that if the sequence $\left\{\epsilon_{n}\right\}$ of parameters satisfies appropriate conditions, then the sequence $\left\{x_{n}\right\}$ generated by (1.13) strongly converges to the unique solution $\tilde{x}=P_{F(T)}(I-A+$ $\gamma f) \tilde{x}$ of the variational inequality

$$
\langle(A-\gamma f) \tilde{x}, x-\tilde{x}\rangle \geq 0, \quad \forall x \in F(T),
$$

which is the optimality condition for the minimization problem

$$
\min _{x \in F(T)} \frac{1}{2}\langle A x, x\rangle-h(x)
$$

where $h$ is a potential function for $\gamma f$ (i.e., $h^{\prime}(x)=\gamma f(x)$ for $x \in H$ ).

In 2010, Yao et al. [9] introduced an iterative algorithm for solving some hierarchical fixed point problem, starting with an arbitrary initial guess $x_{0} \in C$, define a sequence $\left\{x_{n}\right\}$ iteratively by

$$
\begin{gathered}
y_{n}=\beta_{n} S x_{n}+\left(1-\beta_{n}\right) x_{n}, \\
x_{n+1}=P_{C}\left[\alpha_{n} f\left(x_{n}\right)+\left(1-\alpha_{n}\right) T y_{n}\right], \quad \forall n \geq 1 .
\end{gathered}
$$

They proved that if the sequences $\left\{\alpha_{n}\right\}$ and $\left\{\beta_{n}\right\}$ of parameters satisfies appropriate conditions, then the sequence $\left\{x_{n}\right\}$ generated by (1.16) strongly converges to the unique solution $z$ in $H$ of the variational inequality

$$
z \in F(T), \quad\langle(I-f) z, x-z\rangle \geq 0, \quad \forall x \in F(T)
$$


In this paper we will combine the general iterative method (1.13) with the iterative algorithm (1.16) and consider the following iterative algorithm:

$$
\begin{gathered}
y_{n}=\beta_{n} S x_{n}+\left(1-\beta_{n}\right) x_{n} \\
x_{n+1}=P_{C}\left[\alpha_{n} \gamma f\left(x_{n}\right)+\left(I-\alpha_{n} A\right) T y_{n}\right], \quad \forall n \geq 1 .
\end{gathered}
$$

We will prove in Section 3 that if the sequences $\left\{\alpha_{n}\right\}$ and $\left\{\beta_{n}\right\}$ of parameters satisfy appropriate conditions and $\lim _{n \rightarrow \infty}\left(\beta_{n} / \alpha_{n}\right)=\tau \in(0, \infty)$ then the sequence $\left\{x_{n}\right\}$ generated by (1.18) converges strongly to the unique solution $\tilde{x}$ in $H$ of the following variational inequality

$$
\tilde{x} \in F(T), \quad\left\langle\frac{1}{\tau}(A-\gamma f) \tilde{x}+(I-S) \tilde{x}, \quad x-\tilde{x}\right\rangle \geq 0, \quad \forall x \in F(T) .
$$

In particular, if we take $\tau=0$, under suitable difference assumptions on parameter, then the sequence $\left\{x_{n}\right\}$ generated by (1.18) converges strongly to the unique solution $\tilde{x}$ in $H$ of the following variational inequality

$$
\tilde{x} \in F(T), \quad\langle(A-\gamma f) \tilde{x}, x-\tilde{x}\rangle \geq 0, \quad \forall x \in F(T) .
$$

Our results improve and extend the recent results of Yao et al. [9] and some authors. Furthermore, we give an example which supports our main theorem in the last part.

\section{Preliminaries}

This section collects some lemma which can be used in the proofs for the main results in the next section. Some of them are known, others are not hard to derive.

Lemma 2.1 (Browder [10]). Let $H$ be a Hilbert space, $C$ be a closed convex subset of $H$, and $T$ : $C \rightarrow C$ be a nonexpansive mapping with $F(T) \neq \emptyset$. If $\left\{x_{n}\right\}$ is a sequence in $C$ weakly converging to $x$ and if $\left\{(I-T) x_{n}\right\}$ converges strongly to $y$, then $(I-T) x=y$; in particular, if $y=0$ then $x \in F(T)$.

Lemma 2.2. Let $x \in H$ and $z \in C$ be any points. Then one has the following:

(1) That $z=P_{C}[x]$ if and only if there holds the relation:

$$
\langle x-z, y-z\rangle \leq 0, \quad \forall y \in C
$$

(2) That $z=P_{C}[x]$ if and only if there holds the relation:

$$
\|x-z\|^{2} \leq\|x-y\|^{2}-\|y-z\|^{2}, \quad \forall y \in C .
$$


Journal of Applied Mathematics

(3) There holds the relation:

$$
\left\langle P_{C}[x]-P_{C}[y], x-y\right\rangle \geq\left\|P_{C}[x]-P_{C}[y]\right\|^{2}, \quad \forall x, y \in H .
$$

Consequently, $P_{C}$ is nonexpansive and monotone.

Lemma 2.3 (Marino and $\mathrm{Xu}[8]$ ). Let $H$ be a Hilbert space, $C$ be a closed convex subset of $H$, $f: C \rightarrow H$ be a contraction with coefficient $0<\rho<1$, and $T: C \rightarrow C$ be nonexpansive mapping. Let $A$ be a strongly positive linear bounded operator on a Hilbert space $H$ with coefficient $\bar{\gamma}>0$. Then, for $0<\gamma<\bar{\gamma} / \rho$, for $x, y \in C$,

(1) the mapping $(I-f)$ is strongly monotone with coefficient $(1-\rho)$, that is,

$$
\langle x-y,(I-f) x-(I-f) y\rangle \geq(1-\rho)\|x-y\|^{2},
$$

(2) the mapping $(A-\gamma f)$ is strongly monotone with coefficient $\bar{\gamma}-\gamma \rho$ that is

$$
\langle x-y,(A-\gamma f) x-(A-\gamma f) y\rangle \geq(\bar{r}-\gamma \rho)\|x-y\|^{2} .
$$

Lemma 2.4 (Xu [4]). Assume that $\left\{a_{n}\right\}$ is a sequence of nonnegative numbers such that

$$
a_{n+1} \leq\left(1-\gamma_{n}\right) a_{n}+\delta_{n}, \quad \forall n \geq 0,
$$

where $\left\{\gamma_{n}\right\}$ is a sequence in $(0,1)$ and $\left\{\delta_{n}\right\}$ is a sequence in $\mathbb{R}$ such that

(1) $\sum_{n=1}^{\infty} \gamma_{n}=\infty$,

(2) $\lim \sup _{n \rightarrow \infty}\left(\delta_{n} / \gamma_{n}\right) \leq 0$ or $\sum_{n=1}^{\infty}\left|\delta_{n}\right|<\infty$. Then $\lim _{n \rightarrow \infty} a_{n}=0$.

Lemma 2.5 (Marino and Xu [8]). Assume $A$ is a strongly positive linear bounded operator on a Hilbert space $H$ with coefficient $\bar{\gamma}>0$ and $0<\alpha \leq\|A\|^{-1}$. Then $\|I-\alpha A\| \leq 1-\alpha \bar{\gamma}$.

Lemma 2.6 (Acedo and $\mathrm{Xu}[11]$ ). Let $C$ be a closed convex subset of $H$. Let $\left\{x_{n}\right\}$ be a bounded sequence in $H$. Assume that

(1) The weak $\omega$-limit set $\omega_{w}\left(x_{n}\right) \subset C$,

(2) For each $z \in C, \lim _{n \rightarrow \infty}\left\|x_{n}-z\right\|$ exists. Then $\left\{x_{n}\right\}$ is weakly convergent to a point in $C$.

Notation. We use $\rightarrow$ for strong convergence and $\rightarrow$ for weak convergence.

\section{Main Results}

Theorem 3.1. Let $C$ be a nonempty closed convex subset of a real Hilbert space $H$. Let $f: C \rightarrow H$ be a $\rho$-contraction with $\rho \in(0,1)$. Let $S, T: C \rightarrow C$ be two nonexpansive mappings with $F(T) \neq \emptyset$. 
Let $A$ be a strongly positive linear bounded operator on $H$ with coefficient $\bar{\gamma}>0 .\left\{\alpha_{n}\right\}$ and $\left\{\beta_{n}\right\}$ are two sequences in $(0,1)$ and $0<\gamma<\bar{\gamma} / \rho$. Starting with an arbitrary initial guess $x_{0} \in C$ and $\left\{x_{n}\right\}$ is a sequence generated by

$$
\begin{gathered}
y_{n}=\beta_{n} S x_{n}+\left(1-\beta_{n}\right) x_{n}, \\
x_{n+1}=P_{C}\left[\alpha_{n} \gamma f\left(x_{n}\right)+\left(I-\alpha_{n} A\right) T y_{n}\right], \quad \forall n \geq 1 .
\end{gathered}
$$

Suppose that the following conditions are satisfied:

(C1) $\lim _{n \rightarrow \infty} \alpha_{n}=0$ and $\sum_{n=1}^{\infty} \alpha_{n}=\infty$,

(C2) $\lim _{n \rightarrow \infty}\left(\beta_{n} / \alpha_{n}\right)=\tau=0$,

(C3) $\lim _{n \rightarrow \infty}\left(\left|\alpha_{n}-\alpha_{n-1}\right| / \alpha_{n}\right)=0$ and $\lim _{n \rightarrow \infty}\left(\left|\beta_{n}-\beta_{n-1}\right| / \beta_{n}\right)=0$, or

(C4) $\sum_{n=1}^{\infty}\left|\alpha_{n}-\alpha_{n-1}\right|<\infty$ and $\sum_{n=1}^{\infty}\left|\beta_{n}-\beta_{n-1}\right|<\infty$.

Then the sequence $\left\{x_{n}\right\}$ converges strongly to a point $\tilde{x} \in H$, which is the unique solution of the variational inequality:

$$
\tilde{x} \in F(T), \quad\langle(A-\gamma f) \tilde{x}, x-\tilde{x}\rangle \geq 0, \quad \forall x \in F(T) .
$$

Equivalently, one has $P_{F(T)}(I-A+\gamma f) \tilde{x}=\tilde{x}$.

Proof. We first show the uniqueness of a solution of the variational inequality (3.2), which is indeed a consequence of the strong monotonicity of $A-\gamma f$. Suppose $\bar{x} \in F(T)$ and $\tilde{x} \in F(T)$ both are solutions to (3.2), then $\langle(A-\gamma f) \bar{x}, \bar{x}-\tilde{x}\rangle \leq 0$ and $\langle(A-\gamma f) \tilde{x}, \tilde{x}-\bar{x}\rangle \leq 0$. It follows that

$$
\begin{aligned}
\langle(A-\gamma f) \bar{x}, \bar{x}-\tilde{x}\rangle+\langle(A-\gamma f) \tilde{x}, \tilde{x}-\bar{x}\rangle & =\langle(A-\gamma f) \bar{x}, \bar{x}-\tilde{x}\rangle-\langle(A-\gamma f) \tilde{x}, \bar{x}-\tilde{x}\rangle \\
& =\langle(A-\gamma f) \bar{x}-(A-\gamma f) \tilde{x}, \bar{x}-\tilde{x}\rangle
\end{aligned}
$$

The strong monotonicity of $A-\gamma f$ (Lemma 2.3) implies that $\bar{x}=\tilde{x}$ and the uniqueness is proved.

Next, we prove that the sequence $\left\{x_{n}\right\}$ is bounded. Since $\alpha_{n} \rightarrow 0$ and $\lim _{n \rightarrow \infty}\left(\beta_{n} / \alpha_{n}\right)=0$ by condition (C1) and (C2), respectively, we can assume, without loss of generality, that $\alpha_{n}<\|A\|^{-1}$ and $\beta_{n}<\alpha_{n}$ for all $n \geq 1$. Take $u \in F(T)$ and from (3.1), we have

$$
\begin{aligned}
\left\|x_{n+1}-u\right\| & =\left\|P_{C}\left[\alpha_{n} \gamma f\left(x_{n}\right)+\left(I-\alpha_{n} A\right) T y_{n}\right]-P_{C}[u]\right\| \\
& \leq\left\|\alpha_{n} \gamma f\left(x_{n}\right)+\left(I-\alpha_{n} A\right) T y_{n}-u\right\| \\
& \leq \alpha_{n} \gamma\left\|f\left(x_{n}\right)-f(u)\right\|+\alpha_{n}\|\gamma f(u)-A u\|+\left\|\left(I-\alpha_{n} A\right)\left(T y_{n}-u\right)\right\| .
\end{aligned}
$$


Since $\left\|I-\alpha_{n} A\right\| \leq 1-\alpha_{n} \bar{r}$ and by Lemma 2.5 , we note that

$$
\begin{aligned}
& \left\|x_{n+1}-u\right\| \leq \alpha_{n} \gamma\left\|f\left(x_{n}\right)-f(u)\right\|+\alpha_{n}\|\gamma f(u)-A u\|+\left(1-\alpha_{n} \bar{\gamma}\right)\left\|T y_{n}-u\right\| \\
& \leq \alpha_{n} \gamma \rho\left\|x_{n}-u\right\|+\alpha_{n}\|\gamma f(u)-A u\|+\left(1-\alpha_{n} \bar{\gamma}\right)\left\|T y_{n}-T u\right\| \\
& \leq \alpha_{n} \gamma \rho\left\|x_{n}-u\right\|+\alpha_{n}\|\gamma f(u)-A u\|+\left(1-\alpha_{n} \bar{\gamma}\right)\left\|y_{n}-u\right\| \\
& \leq \alpha_{n} \gamma \rho\left\|x_{n}-u\right\|+\alpha_{n}\|\gamma f(u)-A u\| \\
& +\left(1-\alpha_{n} \bar{\gamma}\right)\left[\beta_{n}\left\|S x_{n}-S u\right\|+\beta_{n}\|S u-u\|+\left(1-\beta_{n}\right)\left\|x_{n}-u\right\|\right] \\
& \leq \alpha_{n} \gamma \rho\left\|x_{n}-u\right\|+\alpha_{n}\|\gamma f(u)-A u\| \\
& +\left(1-\alpha_{n} \bar{\gamma}\right)\left[\beta_{n}\left\|x_{n}-u\right\|+\beta_{n}\|S u-u\|+\left(1-\beta_{n}\right)\left\|x_{n}-u\right\|\right] \\
& =\left(1-\alpha_{n}(\bar{\gamma}-\gamma \rho)\right)\left\|x_{n}-u\right\|+\alpha_{n}\|\gamma f(u)-A u\|+\left(1-\alpha_{n} \bar{\gamma}\right) \beta_{n}\|S u-u\| \\
& \leq\left(1-\alpha_{n}(\bar{\gamma}-\gamma \rho)\right)\left\|x_{n}-u\right\|+\alpha_{n}\|\gamma f(u)-A u\|+\beta_{n}\|S u-u\| \\
& \leq\left(1-\alpha_{n}(\bar{\gamma}-\gamma \rho)\right)\left\|x_{n}-u\right\|+\alpha_{n}\|\gamma f(u)-A u\|+\alpha_{n}\|S u-u\| \\
& =\left(1-\alpha_{n}(\bar{r}-\gamma \rho)\right)\left\|x_{n}-u\right\|+\alpha_{n}[\|\gamma f(u)-A u\|+\|S u-u\|] \\
& =\left(1-\alpha_{n}(\bar{\gamma}-\gamma \rho)\right)\left\|x_{n}-u\right\|+\alpha_{n}(\bar{\gamma}-\gamma \rho) \frac{\|\gamma f(u)-A u\|+\|S u-u\|}{(\bar{\gamma}-\gamma \rho)} \text {. }
\end{aligned}
$$

By induction, we can obtain

$$
\left\|x_{n+1}-u\right\| \leq \max \left\{\left\|x_{0}-u\right\|, \frac{\|\gamma f(u)-A u\|+\|S u-u\|}{(\bar{\gamma}-\gamma \rho)}\right\}
$$

which implies that the sequence $\left\{x_{n}\right\}$ is bounded and so are the sequences $\left\{f\left(x_{n}\right)\right\},\left\{S x_{n}\right\}$, and $\left\{A T y_{n}\right\}$.

Set $w_{n}:=\alpha_{n} \gamma f\left(x_{n}\right)+\left(I-\alpha_{n} A\right) T y_{n}, n \geq 1$. We get

$$
\begin{aligned}
\left\|x_{n+1}-x_{n}\right\| & =\left\|P_{C}\left[w_{n+1}\right]-P_{C}\left[w_{n}\right]\right\| \\
& \leq\left\|w_{n+1}-w_{n}\right\| .
\end{aligned}
$$


It follows that

$$
\begin{aligned}
\left\|x_{n+1}-x_{n}\right\| \leq & \left\|\left(\alpha_{n} \gamma f\left(x_{n}\right)+\left(I-\alpha_{n} A\right) T y_{n}\right)-\left(\alpha_{n-1} \gamma f\left(x_{n-1}\right)+\left(I-\alpha_{n-1} A\right) T y_{n-1}\right)\right\| \\
\leq & \alpha_{n} \gamma\left\|f\left(x_{n}\right)-f\left(x_{n-1}\right)\right\|+\left|\alpha_{n}-\alpha_{n-1}\right|\left\|\gamma f\left(x_{n-1}\right)-A T y_{n-1}\right\| \\
& +\left(1-\alpha_{n} \bar{\gamma}\right)\left\|T y_{n}-T y_{n-1}\right\| \\
\leq & \alpha_{n} \gamma \rho\left\|x_{n}-x_{n-1}\right\|+\left|\alpha_{n}-\alpha_{n-1}\right|\left\|\gamma f\left(x_{n-1}\right)-A T y_{n-1}\right\| \\
& +\left(1-\alpha_{n} \bar{\gamma}\right)\left\|y_{n}-y_{n-1}\right\| .
\end{aligned}
$$

By (3.7) and (3.8), we get

$$
\begin{aligned}
\left\|x_{n+1}-x_{n}\right\| \leq & \alpha_{n} \gamma \rho\left\|w_{n}-w_{n-1}\right\|+\left|\alpha_{n}-\alpha_{n-1}\right|\left\|\gamma f\left(x_{n-1}\right)-A T y_{n-1}\right\| \\
& +\left(1-\alpha_{n} \bar{\gamma}\right)\left\|y_{n}-y_{n-1}\right\| .
\end{aligned}
$$

From (3.1), we obtain

$$
\begin{aligned}
\left\|y_{n}-y_{n-1}\right\| & =\left\|\left(\beta_{n} S x_{n}+\left(1-\beta_{n}\right) x_{n}\right)-\left(\beta_{n-1} S x_{n-1}+\left(1-\beta_{n-1}\right) x_{n-1}\right)\right\| \\
& =\left\|\beta_{n}\left(S x_{n}-S x_{n-1}\right)+\left(\beta_{n}-\beta_{n-1}\right)\left(S x_{n-1}-x_{n-1}\right)+\left(1-\beta_{n}\right)\left(x_{n}-x_{n-1}\right)\right\| \\
& \leq\left\|x_{n}-x_{n-1}\right\|+\left|\beta_{n}-\beta_{n-1}\right|\left\|S x_{n-1}-x_{n-1}\right\| \\
& \leq\left\|x_{n}-x_{n-1}\right\|+\left|\beta_{n}-\beta_{n-1}\right| M,
\end{aligned}
$$

where $M$ is a constant such that

$$
\sup _{n \in \mathbb{N}}\left\{\left\|\gamma f\left(x_{n-1}\right)-A T y_{n-1}\right\|+\left\|S x_{n-1}-x_{n-1}\right\|\right\} \leq M
$$

Substituting (3.10) into (3.8) to obtain

$$
\begin{aligned}
\left\|x_{n+1}-x_{n}\right\| \leq & \alpha_{n} \gamma \rho\left\|x_{n}-x_{n-1}\right\|+\left|\alpha_{n}-\alpha_{n-1}\right|\left\|\gamma f\left(x_{n-1}\right)-A T y_{n-1}\right\| \\
& +\left(1-\alpha_{n} \bar{\gamma}\right)\left[\left\|x_{n}-x_{n-1}\right\|+\left|\beta_{n}-\beta_{n-1}\right| M\right] \\
\leq & \alpha_{n} \gamma \rho\left\|x_{n}-x_{n-1}\right\|+\left|\alpha_{n}-\alpha_{n-1}\right| M \\
& +\left(1-\alpha_{n} \bar{\gamma}\right)\left[\left\|x_{n}-x_{n-1}\right\|+\left|\beta_{n}-\beta_{n-1}\right| M\right]
\end{aligned}
$$


Journal of Applied Mathematics

$$
\begin{aligned}
& =\left(1-\alpha_{n}(\bar{\gamma}-\gamma \rho)\right)\left\|x_{n}-x_{n-1}\right\|+M\left[\left|\alpha_{n}-\alpha_{n-1}\right|+\left|\beta_{n}-\beta_{n-1}\right|\right] \\
& \leq\left(1-\alpha_{n}(\bar{\gamma}-\gamma \rho)\right)\left\|w_{n}-w_{n-1}\right\|+M\left[\left|\alpha_{n}-\alpha_{n-1}\right|+\left|\beta_{n}-\beta_{n-1}\right|\right] .
\end{aligned}
$$

At the same time, we can write (3.12) as

$$
\begin{aligned}
\left\|x_{n+1}-x_{n}\right\| & \leq\left(1-\alpha_{n}(\bar{\gamma}-\gamma \rho)\right)\left\|w_{n}-w_{n-1}\right\|+M \alpha_{n}\left[\frac{\left|\alpha_{n}-\alpha_{n-1}\right|}{\alpha_{n}}+\frac{\left|\beta_{n}-\beta_{n-1}\right|}{\alpha_{n}}\right] \\
& \leq\left(1-\alpha_{n}(\bar{\gamma}-\gamma \rho)\right)\left\|w_{n}-w_{n-1}\right\|+M \alpha_{n}\left[\frac{\left|\alpha_{n}-\alpha_{n-1}\right|}{\beta_{n}}+\frac{\left|\beta_{n}-\beta_{n-1}\right|}{\beta_{n}}\right] .
\end{aligned}
$$

From (3.12), (C4), and Lemma 2.5 or from (3.13), (C3), and Lemma 2.5, we can deduce that $\left\|x_{n+1}-x_{n}\right\| \rightarrow 0$, respectively.

From (3.1), we have

$$
\begin{aligned}
\left\|x_{n}-T x_{n}\right\| & \leq\left\|x_{n}-x_{n+1}\right\|+\left\|x_{n+1}-T x_{n}\right\| \\
& =\left\|x_{n}-x_{n+1}\right\|+\left\|P_{C}\left[w_{n}\right]-P_{C}\left[T x_{n}\right]\right\| \\
& \leq\left\|x_{n}-x_{n+1}\right\|+\left\|w_{n}-T x_{n}\right\| \\
& =\left\|x_{n}-x_{n+1}\right\|+\left\|\alpha_{n} \gamma f\left(x_{n}\right)+\left(I-\alpha_{n} A\right) T y_{n}-T x_{n}\right\| \\
& \leq\left\|x_{n}-x_{n+1}\right\|+\alpha_{n}\left\|\gamma f\left(x_{n}\right)-A T x_{n}\right\|+\left(1-\alpha_{n} \bar{\gamma}\right)\left\|T y_{n}-T x_{n}\right\| \\
& \leq\left\|x_{n}-x_{n+1}\right\|+\alpha_{n}\left\|\gamma f\left(x_{n}\right)-A T x_{n}\right\|+\left(1-\alpha_{n} \bar{\gamma}\right)\left\|y_{n}-x_{n}\right\| \\
& =\left\|x_{n}-x_{n+1}\right\|+\alpha_{n}\left\|\gamma f\left(x_{n}\right)-A T x_{n}\right\|+\left(1-\alpha_{n} \bar{\gamma}\right) \beta_{n}\left\|S x_{n}-x_{n}\right\| .
\end{aligned}
$$

Notice that $\alpha_{n} \rightarrow 0, \beta_{n} \rightarrow 0$, and $\left\|x_{n+1}-x_{n}\right\| \rightarrow 0$, so we obtain

$$
\left\|x_{n}-T x_{n}\right\| \longrightarrow 0
$$

Next, we prove

$$
\limsup _{n \rightarrow \infty}\left\langle\gamma f(z)-A z, x_{n}-z\right\rangle \leq 0
$$

where $z=P_{F(T)}(I-A+\gamma f) z$. Since the sequence $\left\{x_{n}\right\}$ is bounded we can take a subsequence $\left\{x_{n_{k}}\right\}$ of $\left\{x_{n}\right\}$ such that

$$
\limsup _{n \rightarrow \infty}\left\langle\gamma f(z)-A z, x_{n}-z\right\rangle=\lim _{k \rightarrow \infty}\left\langle\gamma f(z)-A z, x_{n_{k}}-z\right\rangle
$$


and $x_{n_{k}}-\tilde{x}$. From (3.15) and by Lemma 2.1, it follows that $\tilde{x} \in F(T)$. Hence, by Lemma 2.2(1) that

$$
\begin{aligned}
\limsup _{n \rightarrow \infty}\left\langle\gamma f(z)-A z, x_{n}-z\right\rangle & =\lim _{k \rightarrow \infty}\left\langle\gamma f(z)-A z, x_{n_{k}}-z\right\rangle \\
& =\langle\gamma f(z)-A z, \tilde{x}-z\rangle \\
& =\langle(I-A+\gamma f) z-z, \tilde{x}-z\rangle \\
& \leq 0 .
\end{aligned}
$$

Now, by Lemma 2.2(1), we observe that

$$
\left\langle P_{C}\left[w_{n}\right]-w_{n}, P_{C}\left[w_{n}\right]-z\right\rangle \leq 0,
$$

and so

$$
\begin{aligned}
\left\|x_{n+1}-z\right\|^{2}= & \left\langle P_{C}\left[w_{n}\right]-z, P_{C}\left[w_{n}\right]-z\right\rangle \\
= & \left\langle P_{C}\left[w_{n}\right]-w_{n}, P_{C}\left[w_{n}\right]-z\right\rangle+\left\langle w_{n}-z, P_{C}\left[w_{n}\right]-z\right\rangle \\
\leq & \left\langle w_{n}-z, P_{C}\left[w_{n}\right]-z\right\rangle \\
= & \left\langle\alpha_{n} \gamma f\left(x_{n}\right)+\left(I-\alpha_{n} A\right) T y_{n}-z, x_{n+1}-z\right\rangle \\
\leq & \alpha_{n} \gamma\left\|f\left(x_{n}\right)-f(z)\right\|\left\|x_{n+1}-z\right\|+\alpha_{n}\left\langle\gamma f(z)-A z, x_{n+1}-z\right\rangle \\
& +\left(1-\alpha_{n} \bar{\gamma}\right)\left\|T y_{n}-z\right\|\left\|x_{n+1}-z\right\| \\
\leq & \alpha_{n} \gamma \rho\left\|x_{n}-z\right\|\left\|x_{n+1}-z\right\|+\alpha_{n}\left\langle\gamma f(z)-A z, x_{n+1}-z\right\rangle \\
& +\left(1-\alpha_{n} \bar{\gamma}\right)\left\|y_{n}-z\right\|\left\|x_{n+1}-z\right\| \\
= & \alpha_{n} \gamma \rho\left\|x_{n}-z\right\|\left\|x_{n+1}-z\right\|+\alpha_{n}\left\langle\gamma f(z)-A z, x_{n+1}-z\right\rangle \\
& +\left(1-\alpha_{n} \bar{\gamma}\right)\left\|\beta_{n} S x_{n}+\left(1-\beta_{n}\right) x_{n}-z\right\|\left\|x_{n+1}-z\right\| \\
\leq & \alpha_{n} \gamma \rho\left\|x_{n}-z\right\|\left\|x_{n+1}-z\right\|+\alpha_{n}\left\langle\gamma f(z)-A z, x_{n+1}-z\right\rangle \\
& +\left(1-\alpha_{n} \bar{\gamma}\right)\left[\beta_{n}\left\|S x_{n}-S z\right\|+\beta_{n}\|S z-z\|+\left(1-\beta_{n}\right)\left\|x_{n}-z\right\|\right]\left\|x_{n+1}-z\right\| \\
\leq & \alpha_{n} \gamma \rho\left\|x_{n}-z\right\|\left\|x_{n+1}-z\right\|+\alpha_{n}\left\langle\gamma f(z)-A z, x_{n+1}-z\right\rangle \\
& +\left(1-\alpha_{n} \bar{\gamma}\right)\left[\beta_{n}\left\|x_{n}-z\right\|+\beta_{n}\|S z-z\|+\left(1-\beta_{n}\right)\left\|x_{n}-z\right\|\right]\left\|x_{n+1}-z\right\| \\
& (1)
\end{aligned}
$$


Journal of Applied Mathematics

$$
\begin{aligned}
= & \left(1-\alpha_{n}(\bar{\gamma}-\gamma \rho)\right)\left\|x_{n}-z\right\|\left\|x_{n+1}-z\right\|+\alpha_{n}\left\langle\gamma f(z)-A z, x_{n+1}-z\right\rangle \\
& +\left(1-\alpha_{n} \bar{\gamma}\right) \beta_{n}\|S z-z\|\left\|x_{n+1}-z\right\| \\
\leq & \frac{\left[1-\alpha_{n}(\bar{\gamma}-\gamma \rho)\right]}{2}\left[\left\|x_{n}-z\right\|^{2}+\left\|x_{n+1}-z\right\|^{2}\right]+\alpha_{n}\left\langle\gamma f(z)-A z, x_{n+1}-z\right\rangle \\
& +\left(1-\alpha_{n} \bar{\gamma}\right) \beta_{n}\|S z-z\|\left\|x_{n+1}-z\right\| .
\end{aligned}
$$

Hence, it follows that

$$
\begin{aligned}
\left\|x_{n+1}-z\right\|^{2} \leq & \frac{1-\alpha_{n}(\bar{\gamma}-\gamma \rho)}{1+\alpha_{n}(\bar{\gamma}-\gamma \rho)}\left\|x_{n}-z\right\|^{2}+\frac{2 \alpha_{n}}{1+\alpha_{n}(\bar{\gamma}-\gamma \rho)}\left\langle\gamma f(z)-A z, x_{n+1}-z\right\rangle \\
& +\frac{2\left(1-\alpha_{n} \bar{\gamma}\right) \beta_{n}}{1+\alpha_{n}(\bar{\gamma}-\gamma \rho)}\|S z-z\|\left\|x_{n+1}-z\right\| \\
= & {\left[\frac { 2 \alpha _ { n } ( \overline { \gamma } - \gamma \rho ) } { 1 + \alpha _ { n } ( \overline { \gamma } - \gamma \rho ) ] } \left[\frac{1}{\alpha_{n}(\bar{\gamma}-\gamma \rho)}\left\langle\gamma f(z)-A z, x_{n+1}-z\right\rangle\right.\right.} \\
& \times\left[\begin{array}{c}
1-\frac{\beta_{n}\left(1-\alpha_{n} \bar{\gamma}\right)}{1+\alpha_{n}(\bar{\gamma}-\gamma \rho)}\|S z-z\| x_{n+1}-z \| \\
+\frac{\alpha_{n}(\bar{\gamma}-\gamma \rho)}{\left\|x_{n}-z\right\|^{2} .}
\end{array}\right]
\end{aligned}
$$

We observe that

$$
\limsup _{n \rightarrow \infty}\left[\frac{1}{\alpha_{n}(\bar{\gamma}-\gamma \rho)}\left\langle\gamma f(z)-A z, x_{n+1}-z\right\rangle+\frac{\beta_{n}\left(1-\alpha_{n} \bar{\gamma}\right)}{\alpha_{n}(\bar{\gamma}-\gamma \rho)}\|S z-z\|\left\|x_{n+1}-z\right\|\right] \leq 0 .
$$

Thus, by Lemma 2.4, $x_{n} \rightarrow z$ as $n \rightarrow \infty$. This is completes. 
From Theorem 3.1, we can deduce the following interesting corollary.

Corollary 3.2 (Yao et al. [9]). Let $C$ be a nonempty closed convex subset of a real Hilbert space $H$. Let $f: C \rightarrow H$ be a $\rho$-contraction (possibly nonself) with $\rho \in(0,1)$. Let $S, T: C \rightarrow C$ be two nonexpansive mappings with $F(T) \neq \emptyset .\left\{\alpha_{n}\right\}$ and $\left\{\beta_{n}\right\}$ are two sequences in $(0,1)$. Starting with an arbitrary initial guess $x_{0} \in C$ and $\left\{x_{n}\right\}$ is a sequence generated by

$$
\begin{gathered}
y_{n}=\beta_{n} S x_{n}+\left(1-\beta_{n}\right) x_{n} \\
x_{n+1}=P_{C}\left[\alpha_{n} f\left(x_{n}\right)+\left(1-\alpha_{n}\right) T y_{n}\right], \quad \forall n \geq 1 .
\end{gathered}
$$

Suppose that the following conditions are satisfied:

(C1) $\lim _{n \rightarrow \infty} \alpha_{n}=0$ and $\sum_{n=1}^{\infty} \alpha_{n}=\infty$,

(C2) $\lim _{n \rightarrow \infty}\left(\beta_{n} / \alpha_{n}\right)=0$,

(C3) $\lim _{n \rightarrow \infty}\left(\left|\alpha_{n}-\alpha_{n-1}\right| / \alpha_{n}\right)=0$ and $\lim _{n \rightarrow \infty}\left(\left|\beta_{n}-\beta_{n-1}\right| / \beta_{n}\right)=0$, or

(C4) $\sum_{n=1}^{\infty}\left|\alpha_{n}-\alpha_{n-1}\right|<\infty$ and $\sum_{n=1}^{\infty}\left|\beta_{n}-\beta_{n-1}\right|<\infty$.

Then the sequence $\left\{x_{n}\right\}$ converges strongly to a point $\tilde{x} \in H$, which is the unique solution of the variational inequality:

$$
\tilde{x} \in F(T), \quad\langle(I-f) \tilde{x}, x-\tilde{x}\rangle \geq 0, \quad \forall x \in F(T) .
$$

Equivalently, one has $P_{F(T)}(f) \tilde{x}=\tilde{x}$. In particular, if one takes $f=0$, then the sequence $\left\{x_{n}\right\}$ converges in norm to the Minimum norm fixed point $\tilde{x}$ of $T$, namely, the point $\tilde{x}$ is the unique solution to the quadratic minimization problem:

$$
z=\arg \min _{x \in F(T)}\|x\|^{2}
$$

Proof. As a matter of fact, if we take $A=I$ and $\gamma=1$ in Theorem 3.1. This completes the proof.

Under different conditions on data we obtain the following result.

Theorem 3.3. Let $C$ be a nonempty closed convex subset of a real Hilbert space $H$. Let $f: C \rightarrow$ $H$ be a $\rho$-contraction (possibly nonself) with $\rho \in(0,1)$. Let $S, T: C \rightarrow C$ be two nonexpansive mappings with $F(T) \neq \emptyset$. Let $A$ be a strongly positive linear bounded operator on a Hilbert space $H$ with coefficient $\bar{\gamma}>0$ and $0<\gamma<\bar{\gamma} / \rho .\left\{\alpha_{n}\right\}$ and $\left\{\beta_{n}\right\}$ are two sequences in $(0,1)$. Starting with an arbitrary initial guess $x_{0} \in C$ and $\left\{x_{n}\right\}$ is a sequence generated by

$$
\begin{gathered}
y_{n}=\beta_{n} S x_{n}+\left(1-\beta_{n}\right) x_{n} \\
x_{n+1}=P_{C}\left[\alpha_{n} \gamma f\left(x_{n}\right)+\left(I-\alpha_{n} A\right) T y_{n}\right], \quad \forall n \geq 1 .
\end{gathered}
$$


Journal of Applied Mathematics

Suppose that the following conditions are satisfied:

(C1) $\lim _{n \rightarrow \infty} \alpha_{n}=0$ and $\sum_{n=1}^{\infty} \alpha_{n}=\infty$,

(C2) $\lim _{n \rightarrow \infty}\left(\beta_{n} / \alpha_{n}\right)=\tau \in(0, \infty)$,

(C5) $\lim _{n \rightarrow \infty}\left(\left(\left|\alpha_{n}-\alpha_{n-1}\right|+\left|\beta_{n}-\beta_{n-1}\right|\right) / \alpha_{n} \beta_{n}\right)=0$,

(C6) there exists a constant $K>0$ such that $\left(1 / \alpha_{n}\right)\left|1 / \beta_{n}-1 / \beta_{n-1}\right| \leq K$.

Then the sequence $\left\{x_{n}\right\}$ converges strongly to a point $\tilde{x} \in H$, which is the unique solution of the variational inequality:

$$
\tilde{x} \in F(T), \quad\left\langle\frac{1}{\tau}(A-\gamma f) \tilde{x}+(I-S) \tilde{x}, x-\tilde{x}\right\rangle \geq 0, \quad \forall x \in F(T) .
$$

Proof. First of all, we show that (3.27) has the unique solution. Indeed, let $\bar{x}$ and $\tilde{x}$ be two solutions. Then

$$
\langle(A-\gamma f) \tilde{x}, \tilde{x}-\bar{x}\rangle \leq \tau\langle(I-S) \tilde{x}, \bar{x}-\tilde{x}\rangle .
$$

Analogously, we have

$$
\langle(A-\gamma f) \bar{x}, \bar{x}-\tilde{x}\rangle \leq \tau\langle(I-S) \bar{x}, \tilde{x}-\bar{x}\rangle \text {. }
$$

Adding (3.28) and (3.29), by Lemma 2.3, we obtain

$$
\begin{aligned}
(\bar{\gamma}-\gamma \rho)\|\tilde{x}-\bar{x}\|^{2} & \leq\langle(A-\gamma f) \tilde{x}-(A-\gamma f) \bar{x}, \tilde{x}-\bar{x}\rangle \\
& \leq-\tau\langle(I-S) \tilde{x}-(I-S) \bar{x}, \tilde{x}-\bar{x}\rangle \\
& \leq 0,
\end{aligned}
$$


and so $\tilde{x}=\bar{x}$. From (C2), we can assume, without loss of generality, that $\beta_{n} \leq(\tau+1) \alpha_{n}$ for all $n \geq 1$. By a similar argument in Theorem 3.1, we have

$$
\begin{aligned}
\left\|x_{n+1}-u\right\| \leq & \alpha_{n} \gamma \rho\left\|x_{n}-u\right\|+\alpha_{n}\|\gamma f(u)-A u\| \\
& +\left(1-\alpha_{n} \bar{\gamma}\right)\left[\left\|x_{n}-u\right\|+\beta_{n}\|S u-u\|+\left(1-\beta_{n}\right)\left\|x_{n}-u\right\|\right] \\
= & \left(1-\alpha_{n}(\bar{\gamma}-\gamma \rho)\right)\left\|x_{n}-u\right\|+\alpha_{n}\|\gamma f(u)-A u\|+\left(1-\alpha_{n} \bar{\gamma}\right) \beta_{n}\|S u-u\| \\
\leq & \left(1-\alpha_{n}(\bar{\gamma}-\gamma \rho)\right)\left\|x_{n}-u\right\|+\alpha_{n}\|\gamma f(u)-A u\|+\beta_{n}\|S u-u\| \\
\leq & \left(1-\alpha_{n}(\bar{\gamma}-\gamma \rho)\right)\left\|x_{n}-u\right\|+\alpha_{n}\|\gamma f(u)-A u\|+(\tau+1) \alpha_{n}\|S u-u\| \\
= & \left(1-\alpha_{n}(\bar{r}-\gamma \rho)\right)\left\|x_{n}-u\right\|+\alpha_{n}[\|\gamma f(u)-A u\|+(\tau+1)\|S u-u\|] \\
= & \left(1-\alpha_{n}(\bar{\gamma}-\gamma \rho)\right)\left\|x_{n}-u\right\|+\alpha_{n}(\bar{r}-\gamma \rho) \frac{\|\gamma f(u)-A u\|+(\tau+1)\|S u-u\|}{(\bar{\gamma}-\gamma \rho)} .
\end{aligned}
$$

By induction, we obtain

$$
\left\|x_{n}-u\right\| \leq \max \left\{\left\|x_{0}-u\right\|, \frac{1}{\bar{\gamma}-\gamma \rho}[\|\gamma f(u)-A u\|+(\tau+1)\|S u-u\|]\right\},
$$

which implies that the sequence $\left\{x_{n}\right\}$ is bounded. Since (C5) implies (C4) then, from Theorem 3.1, we can deduce $\left\|x_{n+1}-x_{n}\right\| \rightarrow 0$.

From (3.1), we note that

$$
\begin{aligned}
x_{n+1} & =P_{C}\left[w_{n}\right]-w_{n}+w_{n}+y_{n}-y_{n} \\
& =P_{C}\left[w_{n}\right]-w_{n}+\alpha_{n} \gamma f\left(x_{n}\right)+\left(T y_{n}-y_{n}\right)+\left(y_{n}-\alpha_{n} A T y_{n}\right) .
\end{aligned}
$$

Hence, it follows that

$$
\begin{aligned}
x_{n}-x_{n+1} & =\left(w_{n}-P_{C}\left[w_{n}\right]\right)+\alpha_{n}\left(A x_{n}-\gamma f\left(x_{n}\right)\right)+\left(y_{n}-T y_{n}\right)+\left(x_{n}-y_{n}\right)+\alpha_{n}\left(A T y_{n}-A x_{n}\right) \\
& =\left(w_{n}-P_{C}\left[w_{n}\right]\right)+\alpha_{n}(A-\gamma f) x_{n}+(I-T) y_{n}+\beta_{n}(I-S) x_{n}+\alpha_{n} A\left(T y_{n}-x_{n}\right),
\end{aligned}
$$


and so

$$
\begin{aligned}
\frac{x_{n}-x_{n+1}}{\left(1-\alpha_{n}\right) \beta_{n}}= & \frac{1}{\left(1-\alpha_{n}\right) \beta_{n}}\left(w_{n}-P_{C}\left[w_{n}\right]\right)+\frac{\alpha_{n}}{\left(1-\alpha_{n}\right) \beta_{n}}(A-\gamma f) x_{n}+\frac{1}{\left(1-\alpha_{n}\right) \beta_{n}}(I-T) y_{n} \\
& +\frac{1}{\left(1-\alpha_{n}\right)}(I-S) x_{n}+\frac{\alpha_{n}}{\left(1-\alpha_{n}\right) \beta_{n}} A\left(T y_{n}-x_{n}\right) .
\end{aligned}
$$

Set $v_{n}:=\left(x_{n}-x_{n+1}\right) /\left(1-\alpha_{n}\right) \beta_{n}$. Then, we have

$$
\begin{aligned}
v_{n}= & \frac{1}{\left(1-\alpha_{n}\right) \beta_{n}}\left(w_{n}-P_{C}\left[w_{n}\right]\right)+\frac{\alpha_{n}}{\left(1-\alpha_{n}\right) \beta_{n}}(A-\gamma f) x_{n}+\frac{1}{\left(1-\alpha_{n}\right) \beta_{n}}(I-T) y_{n} \\
& +\frac{1}{\left(1-\alpha_{n}\right)}(I-S) x_{n}+\frac{\alpha_{n}}{\left(1-\alpha_{n}\right) \beta_{n}} A\left(T y_{n}-x_{n}\right) .
\end{aligned}
$$

From (3.12) in Theorem 3.1 and (C6), we obtain

$$
\begin{aligned}
\frac{\left\|x_{n+1}-x_{n}\right\|}{\beta_{n}} \leq & \left(1-\alpha_{n}(\bar{\gamma}-\gamma \rho)\right) \frac{\left\|x_{n}-x_{n-1}\right\|}{\beta_{n}}+M\left[\frac{\left|\alpha_{n}-\alpha_{n-1}\right|}{\beta_{n}}+\frac{\left|\beta_{n}-\beta_{n-1}\right|}{\beta_{n}}\right] \\
= & \left(1-\alpha_{n}(\bar{\gamma}-\gamma \rho)\right) \frac{\left\|x_{n}-x_{n-1}\right\|}{\beta_{n}}+\left(1-\alpha_{n}(\bar{\gamma}-\gamma \rho)\right) \frac{\left\|x_{n}-x_{n-1}\right\|}{\beta_{n-1}} \\
& -\left(1-\alpha_{n}(\bar{\gamma}-\gamma \rho)\right) \frac{\left\|x_{n}-x_{n-1}\right\|}{\beta_{n-1}}+M\left[\frac{\left|\alpha_{n}-\alpha_{n-1}\right|}{\beta_{n}}+\frac{\left|\beta_{n}-\beta_{n-1}\right|}{\beta_{n}}\right] \\
= & \left(1-\alpha_{n}(\bar{\gamma}-\gamma \rho)\right) \frac{\left\|x_{n}-x_{n-1}\right\|}{\beta_{n-1}}+\left(1-\alpha_{n}(\bar{\gamma}-\gamma \rho)\right)\left\|x_{n}-x_{n-1}\right\|\left[\frac{1}{\beta_{n}}-\frac{1}{\beta_{n-1}}\right] \\
& +M\left[\frac{\left|\alpha_{n}-\alpha_{n-1}\right|}{\beta_{n}}+\frac{\left|\beta_{n}-\beta_{n-1}\right|}{\beta_{n}}\right] \\
\leq & \left(1-\alpha_{n}(\bar{\gamma}-\gamma \rho)\right) \frac{\left\|x_{n}-x_{n-1}\right\|}{\beta_{n-1}}+\left\|x_{n}-x_{n-1}\right\|\left|\frac{1}{\beta_{n}}-\frac{1}{\beta_{n-1} \mid}\right| \\
& +M\left[\frac{\left|\alpha_{n}-\alpha_{n-1}\right|}{\beta_{n}}+\frac{\left|\beta_{n}-\beta_{n-1}\right|}{\beta_{n}}\right] \\
\leq & \left(1-\alpha_{n}(\bar{\gamma}-\gamma \rho)\right) \frac{\left\|x_{n}-x_{n-1}\right\|}{\beta_{n-1}}+\alpha_{n} K\left\|x_{n}-x_{n-1}\right\| \\
& +M\left[\frac{\left|\alpha_{n}-\alpha_{n-1}\right|}{\beta_{n}}+\frac{\left|\beta_{n}-\beta_{n-1}\right|}{\beta_{n}}\right]
\end{aligned}
$$




$$
\begin{aligned}
\leq & \left(1-\alpha_{n}(\bar{\gamma}-\gamma \rho)\right) \frac{\left\|w_{n}-w_{n-1}\right\|}{\beta_{n-1}}+\alpha_{n} K\left\|x_{n}-x_{n-1}\right\| \\
& +M\left[\frac{\left|\alpha_{n}-\alpha_{n-1}\right|}{\beta_{n}}+\frac{\left|\beta_{n}-\beta_{n-1}\right|}{\beta_{n}}\right] .
\end{aligned}
$$

This together with Lemma 2.4 and (C2) imply that

$$
\lim _{n \rightarrow \infty} \frac{\left\|x_{n+1}-x_{n}\right\|}{\beta_{n}}=\lim _{n \rightarrow \infty} \frac{\left\|w_{n+1}-w_{n}\right\|}{\beta_{n}}=\lim _{n \rightarrow \infty} \frac{\left\|w_{n+1}-w_{n}\right\|}{\alpha_{n}}=0 .
$$

From (3.36), for $z \in F(T)$, we have

$$
\begin{aligned}
\left\langle v_{n}, x_{n}-z\right\rangle= & \frac{1}{\left(1-\alpha_{n}\right) \beta_{n}}\left\langle w_{n}-P_{C}\left[w_{n}\right], P_{C}\left[w_{n-1}\right]-z\right\rangle+\frac{\alpha_{n}}{\left(1-\alpha_{n}\right) \beta_{n}}\left\langle(A-\gamma f) x_{n}, x_{n}-z\right\rangle \\
& +\frac{1}{\left(1-\alpha_{n}\right) \beta_{n}}\left\langle(I-T) y_{n}, x_{n}-z\right\rangle+\frac{1}{\left(1-\alpha_{n}\right)}\left\langle(I-S) x_{n}, x_{n}-z\right\rangle \\
& +\frac{\alpha_{n}}{\left(1-\alpha_{n}\right) \beta_{n}}\left\langle A\left(T y_{n}-x_{n}\right), x_{n}-z\right\rangle \\
= & \frac{1}{\left(1-\alpha_{n}\right) \beta_{n}}\left\langle w_{n}-P_{C}\left[w_{n}\right], P_{C}\left[w_{n}\right]-z\right\rangle \\
& +\frac{1}{\left(1-\alpha_{n}\right) \beta_{n}}\left\langle w_{n}-P_{C}\left[w_{n}\right], P_{C}\left[w_{n-1}\right]-P_{C}\left[w_{n}\right]\right\rangle \\
& +\frac{\alpha_{n}}{\left(1-\alpha_{n}\right) \beta_{n}}\left\langle(A-\gamma f) x_{n}-(A-\gamma f) z, x_{n}-z\right\rangle+\frac{\alpha_{n}}{\left(1-\alpha_{n}\right) \beta_{n}}\left\langle(A-\gamma f) z, x_{n}-z\right\rangle \\
& +\frac{1}{\left(1-\alpha_{n}\right)}\left\langle(I-S) x_{n}-(I-S) z, x_{n}-z\right\rangle+\frac{1}{\left(1-\alpha_{n}\right)}\left\langle(I-S) z, x_{n}-z\right\rangle \\
& +\frac{1}{\left(1-\alpha_{n}\right) \beta_{n}}\left\langle(I-T) y_{n}, x_{n}-z\right\rangle+\frac{\alpha_{n}}{\left(1-\alpha_{n}\right) \beta_{n}}\left\langle A\left(T y_{n}-x_{n}\right), x_{n}-z\right\rangle .
\end{aligned}
$$

By Lemmas 2.2 and 2.3, we obtain

$$
\begin{aligned}
\left\langle v_{n}, x_{n}-z\right\rangle \geq & \frac{1}{\left(1-\alpha_{n}\right) \beta_{n}}\left\langle w_{n}-P_{C}\left[w_{n}\right], P_{C}\left[w_{n-1}\right]-P_{C}\left[w_{n}\right]\right\rangle+\frac{(\bar{\gamma}-\gamma \rho) \alpha_{n}}{\left(1-\alpha_{n}\right) \beta_{n}}\left\|x_{n}-z\right\|^{2} \\
& +\frac{\alpha_{n}}{\left(1-\alpha_{n}\right) \beta_{n}}\left\langle(A-\gamma f) z, x_{n}-z\right\rangle+\frac{1}{\left(1-\alpha_{n}\right)}\left\langle(I-S) z, x_{n}-z\right\rangle \\
& +\frac{1}{\left(1-\alpha_{n}\right) \beta_{n}}\left\langle(I-T) y_{n}, x_{n}-z\right\rangle+\frac{\alpha_{n}}{\left(1-\alpha_{n}\right) \beta_{n}}\left\langle A\left(T y_{n}-x_{n}\right), x_{n}-z\right\rangle .
\end{aligned}
$$


Now, we observe that

$$
\begin{aligned}
\left\|x_{n}-z\right\|^{2} \leq & \frac{\left(1-\alpha_{n}\right) \beta_{n}}{(\bar{\gamma}-\gamma \rho) \alpha_{n}}\left\langle v, x_{n}-z\right\rangle-\frac{\beta_{n}}{(\bar{\gamma}-\gamma \rho) \alpha_{n}}\left\langle(I-S) z, x_{n}-z\right\rangle \\
& -\frac{1}{(\bar{\gamma}-\gamma \rho)}\left\langle(A-\gamma f) z, x_{n}-z\right\rangle-\frac{1}{(\bar{\gamma}-\gamma \rho) \alpha_{n}}\left\langle(I-T) y_{n}, x_{n}-z\right\rangle \\
& -\frac{1}{(\bar{\gamma}-\gamma \rho)}\left\langle A\left(T y_{n}-x_{n}\right), x_{n}-z\right\rangle \\
& -\frac{1}{(\bar{\gamma}-\gamma \rho) \alpha_{n}}\left\langle w_{n}-P_{C}\left[w_{n}\right], P_{C}\left[w_{n-1}\right]-P_{C}\left[w_{n}\right]\right\rangle \\
\leq & \frac{\left(1-\alpha_{n}\right) \beta_{n}}{(\bar{\gamma}-\gamma \rho) \alpha_{n}}\left\langle v, x_{n}-z\right\rangle-\frac{\beta_{n}}{(\bar{\gamma}-\gamma \rho) \alpha_{n}}\left\langle(I-S) z, x_{n}-z\right\rangle \\
& -\frac{1}{(\bar{\gamma}-\gamma \rho)}\left\langle(A-\gamma f) z, x_{n}-z\right\rangle-\frac{1}{(\bar{\gamma}-\gamma \rho) \alpha_{n}}\left\langle(I-T) y_{n}, x_{n}-z\right\rangle \\
& -\frac{1}{(\bar{\gamma}-\gamma \rho)}\left\langle A\left(T y_{n}-x_{n}\right), x_{n}-z\right\rangle+\frac{\left\|w_{n}-w_{n-1}\right\|}{(\bar{\gamma}-\gamma \rho)}\left\|w_{n}-P_{C}\left[w_{n}\right]\right\| .
\end{aligned}
$$

From (C1) and (C2), we have $\beta_{n} \rightarrow 0$. Hence, from (3.1), we deduce $\left\|y_{n}-x_{n}\right\| \rightarrow 0$ and $\left\|x_{n+1}-T y_{n}\right\| \longrightarrow 0$. Therefore,

$$
\left\|y_{n}-T y_{n}\right\| \leq\left\|y_{n}-x_{n}\right\|+\left\|x_{n}-x_{n+1}\right\|+\left\|x_{n+1}-T y_{n}\right\| \rightarrow 0
$$

Since $v_{n} \rightarrow 0,(I-T) y_{n} \rightarrow 0, A\left(T y_{n}-x_{n}\right) \rightarrow 0$, and $\left\|w_{n}-w_{n-1}\right\| /(\bar{\gamma}-\gamma \rho) \rightarrow 0$, every weak cluster point of $\left\{x_{n}\right\}$ is also a strong cluster point. Note that the sequence $\left\{x_{n}\right\}$ is bounded, thus there exists a subsequence $\left\{x_{n_{k}}\right\}$ converging to a point $\tilde{x} \in H$. For all $z \in F(T)$, it follows from (3.39) that

$$
\begin{aligned}
& \left\langle(A-\gamma f) x_{n_{k}}, x_{n_{k}}-z\right\rangle \\
= & \frac{\left(1-\alpha_{n_{k}}\right) \beta_{n_{k}}}{\alpha_{n_{k}}}\left\langle v_{n_{k}}, x_{n_{k}}-z\right\rangle-\frac{1}{\alpha_{n_{k}}}\left\langle(I-T) y_{n_{k}}, x_{n_{k}}-z\right\rangle-\frac{\beta_{n_{k}}}{\alpha_{n_{k}}}\left\langle(I-S) x_{n_{k}}, x_{n_{k}}-z\right\rangle \\
& -\left\langle A\left(T y_{n_{k}}-x_{n_{k}}\right), x_{n_{k}}-z\right\rangle-\frac{1}{\alpha_{n_{k}}}\left\langle w_{n_{k}}-P_{C}\left[w_{n_{k}}\right], P_{C}\left[w_{n_{k-1}}\right]-z\right\rangle
\end{aligned}
$$




$$
\begin{aligned}
\leq & \frac{\left(1-\alpha_{n_{k}}\right) \beta_{n_{k}}}{\alpha_{n_{k}}}\left\langle v_{n_{k}}, x_{n_{k}}-z\right\rangle-\frac{1}{\alpha_{n_{k}}}\left\langle(I-T) y_{n_{k}}, x_{n_{k}}-z\right\rangle-\frac{\beta_{n_{k}}}{\alpha_{n_{k}}}\left\langle(I-S) x_{n_{k}}, x_{n_{k}}-z\right\rangle \\
& -\left\langle A\left(T y_{n_{k}}-x_{n_{k}}\right), x_{n_{k}}-z\right\rangle-\frac{1}{\alpha_{n_{k}}}\left\langle w_{n_{k}}-P_{C}\left[w_{n_{k}}\right], P_{C}\left[w_{n_{k-1}}\right]-P_{C}\left[w_{n_{k}}\right]\right\rangle \\
& -\left\langle A\left(T y_{n_{k}}-x_{n_{k}}\right), x_{n_{k}}-z\right\rangle-\frac{1}{\alpha_{n_{k}}}\left\langle w_{n_{k}}-P_{C}\left[w_{n_{k}}\right], P_{C}\left[w_{n_{k-1}}\right]-z\right\rangle \\
\leq & \frac{\left(1-\alpha_{n_{k}}\right) \beta_{n_{k}}}{\alpha_{n_{k}}}\left\langle v_{n_{k}}, x_{n_{k}}-z\right\rangle-\frac{1}{\alpha_{n_{k}}}\left\langle(I-T) y_{n_{k}}, x_{n_{k}}-z\right\rangle-\frac{\beta_{n_{k}}}{\alpha_{n_{k}}}\left\langle(I-S) x_{n_{k}}, x_{n_{k}}-z\right\rangle \\
& -\left\langle A\left(T y_{n_{k}}-x_{n_{k}}\right), x_{n_{k}}-z\right\rangle+\frac{\left\|w_{n_{k}}-w_{n_{k-1}}\right\|}{\alpha_{n_{k}}}\left\|w_{n_{k}}-P_{C}\left[w_{n_{k}}\right]\right\| .
\end{aligned}
$$

Letting $k \rightarrow \infty$, we obtain

$$
\langle(A-\gamma f) \tilde{x}, \tilde{x}-z\rangle \leq-\tau\langle(I-S) \tilde{x}, \tilde{x}-z\rangle, \quad \forall z \in F(T) .
$$

By Lemma 2.6 and (3.27) having the unique solution, it follows that $\omega_{w}\left(x_{n}\right)=\{\tilde{x}\}$. Therefore, $x_{n} \rightarrow \tilde{x}$ as $n \rightarrow \infty$. This completes the proof.

From Theorem 3.3, we can deduce the following interesting corollary.

Corollary 3.4 (Yao et al. [9]). Let $C$ be a nonempty closed convex subset of a real Hilbert space $H$. Let $f: C \rightarrow H$ be a $\rho$-contraction (possibly nonself) with $\rho \in(0,1)$. Let $S, T: C \rightarrow C$ be two nonexpansive mappings with $F(T) \neq \emptyset .\left\{\alpha_{n}\right\}$ and $\left\{\beta_{n}\right\}$ are two sequences in $(0,1)$ Starting with an arbitrary initial guess $x_{0} \in C$ and $\left\{x_{n}\right\}$ is a sequence generated by

$$
\begin{gathered}
y_{n}=\beta_{n} S x_{n}+\left(1-\beta_{n}\right) x_{n}, \\
x_{n+1}=P_{C}\left[\alpha_{n} f\left(x_{n}\right)+\left(1-\alpha_{n}\right) T y_{n}\right], \quad \forall n \geq 1 .
\end{gathered}
$$

Suppose that the following conditions are satisfied:

(C1) $\lim _{n \rightarrow \infty} \alpha_{n}=0$ and $\sum_{n=1}^{\infty} \alpha_{n}=\infty$,

(C2) $\lim _{n \rightarrow \infty}\left(\beta_{n} / \alpha_{n}\right)=\tau \in(0, \infty)$,

(C5) $\lim _{n \rightarrow \infty}\left(\left(\left|\alpha_{n}-\alpha_{n-1}\right|+\left|\beta_{n}-\beta_{n-1}\right|\right) / \alpha_{n} \beta_{n}\right)=0$,

(C6) there exists a constant $K>0$ such that $\left(1 / \alpha_{n}\right)\left|1 / \beta_{n}-1 / \beta_{n-1}\right| \leq K$.

Then the sequence $\left\{x_{n}\right\}$ converges strongly to a point $\tilde{x} \in H$, which is the unique solution of the variational inequality:

$$
\tilde{x} \in F(T), \quad\left\langle\frac{1}{\tau}(I-f) \tilde{x}+(I-S) \tilde{x}, x-\tilde{x}\right\rangle \geq 0, \quad \forall x \in F(T) .
$$


Proof. As a matter of fact, if we take $A=I$ and $\gamma=1$ in Theorem 3.3 then this completes the proof.

Corollary 3.5 (Yao et al. [9]). Let $C$ be a nonempty closed convex subset of a real Hilbert space $H$. Let $S, T: C \rightarrow C$ be two nonexpansive mappings with $F(T) \neq \emptyset .\left\{\alpha_{n}\right\}$ and $\left\{\beta_{n}\right\}$ are two sequences in $(0,1)$. Starting with an arbitrary initial guess $x_{0} \in C$ and suppose $\left\{x_{n}\right\}$ is a sequence generated by

$$
\begin{gathered}
y_{n}=\beta_{n} S x_{n}+\left(1-\beta_{n}\right) x_{n}, \\
x_{n+1}=P_{C}\left[\left(1-\alpha_{n}\right) T y_{n}\right], \quad \forall n \geq 1 .
\end{gathered}
$$

Suppose that the following conditions are satisfied:

(C1) $\lim _{n \rightarrow \infty} \alpha_{n}=0$ and $\sum_{n=1}^{\infty} \alpha_{n}=\infty$,

(C2) $\lim _{n \rightarrow \infty}\left(\beta_{n} / \alpha_{n}\right)=1$,

(C5) $\lim _{n \rightarrow \infty}\left(\left(\left|\alpha_{n}-\alpha_{n-1}\right|+\left|\beta_{n}-\beta_{n-1}\right|\right) / \alpha_{n} \beta_{n}\right)=0$,

(C6) there exists a constant $K>0$ such that $\left(1 / \alpha_{n}\right)\left|1 / \beta_{n}-1 / \beta_{n-1}\right| \leq K$.

Then the sequence $\left\{x_{n}\right\}$ converges strongly to a point $\tilde{x} \in H$, which is the unique solution of the variational inequality:

$$
\tilde{x} \in F(T), \quad\left\langle\left(I-\frac{S}{2}\right) \tilde{x}, x-\tilde{x}\right\rangle \geq 0, \quad \forall x \in F(T) .
$$

Proof. As a matter of fact, if we take $A=I, f=0$, and $\gamma=1$ in Theorem 3.3 then this is completes the proof.

Remark 3.6. Prototypes for the iterative parameters are, for example, $\alpha_{n}=n^{-\theta}$ and $\beta_{n}=n^{-\omega}$ (with $\theta, \omega>0$ ). Since $\left|\alpha_{n}-\alpha_{n-1}\right| \approx n^{-\theta}$ and $\left|\beta_{n}-\beta_{n-1}\right| \approx n^{-\omega}$, it is not difficult to prove that (C5) is satisfied for $0<\theta, \omega<1$ and (C6) is satisfied if $\theta+\omega \leq 1$.

Remark 3.7. Our results improve and extend the results of Yao et al. [9] by taking $A=I$ and $r=1$ in Theorems 3.1 and 3.3.

The following is an example to support Theorem 3.3.

Example 3.8. Let $H=\mathbb{R}, \mathbb{C}=[-1 / 4,1 / 4], T=I, S=-I, A=I, f(x)=x^{2}, P_{C}=I, \beta_{n}=$ $1 / \sqrt{n}, \alpha_{n}=1 / \sqrt{n}$ for every $n \in \mathbb{N}$, we have $\tau=1$ and choose $\bar{\gamma}=1 / 2, \rho=1 / 3$ and $\gamma=1$. Then $\left\{x_{n}\right\}$ is the sequence

$$
x_{n+1}=\frac{x_{n}^{2}}{\sqrt{n}}+\left(1-\frac{1}{\sqrt{n}}\right)\left(1-\frac{2}{\sqrt{n}}\right) x_{n}
$$

and $x_{n} \rightarrow \tilde{x}=0$ as $n \rightarrow \infty$, where $\tilde{x}=0$ is the unique solution of the variational inequality

$$
\tilde{x} \in F(T)=\left[-\frac{1}{4}, \frac{1}{4}\right], \quad\left\langle\left(3 \tilde{x}-\tilde{x}^{2}\right), x-\tilde{x}\right\rangle \geq 0, \quad \forall x \in F(T)=\left[-\frac{1}{4}, \frac{1}{4}\right] .
$$




\section{Acknowledgments}

The authors would like to thank the National Research University Project of Thailand's Office of the Higher Education Commission under the Project NRU-CSEC no. 55000613 for financial support.

\section{References}

[1] F. Deutsch and I. Yamada, "Minimizing certain convex functions over the intersection of the fixed point sets of nonexpansive mappings," Numerical Functional Analysis and Optimization, vol. 19, no. 1-2, pp. 33-56, 1998.

[2] I. Yamada, N. Ogura, Y. Yamashita, and K. Sakaniwa, "Quadratic optimization of fixed points of nonexpansive mappings in Hilbert space," Numerical Functional Analysis and Optimization, vol. 19, no. 1-2, pp. 165-190, 1998.

[3] I. Yamada, "The hybrid steepest descent method for the variational inequality problem over the intersection of fixed point sets of nonexpansive mappings," in Inherently Parallel Algorithms in Feasibility and Optimization and their Applications, D. Butnariu, Y. Censor, and S. Reich, Eds., pp. 473504, Elsevier, 2001.

[4] H.-K. Xu, "Iterative algorithms for nonlinear operators," Journal of the London Mathematical Society, vol. 66, no. 1, pp. 240-256, 2002.

[5] H. K. Xu, "An iterative approach to quadratic optimization," Journal of Optimization Theory and Applications, vol. 116, no. 3, pp. 659-678, 2003.

[6] A. Moudafi, "Viscosity approximation methods for fixed-points problems," Journal of Mathematical Analysis and Applications, vol. 241, no. 1, pp. 46-55, 2000.

[7] H.-K. Xu, "Viscosity approximation methods for nonexpansive mappings," Journal of Mathematical Analysis and Applications, vol. 298, no. 1, pp. 279-291, 2004.

[8] G. Marino and H.-K. Xu, "A general iterative method for nonexpansive mappings in Hilbert spaces," Journal of Mathematical Analysis and Applications, vol. 318, no. 1, pp. 43-52, 2006.

[9] Y. Yao, Y. J. Cho, and Y.-C. Liou, "Iterative algorithms for hierarchical fixed points problems and variational inequalities," Mathematical and Computer Modelling, vol. 52, no. 9-10, pp. 1697-1705, 2010.

[10] F. E. Browder, "Nonlinear operators and nonlinear equations of evolution in Banach spaces," in Proceedings of Symposia in Pure Mathematics, vol. 18, pp. 78-81, American Mathematical Society, 1976.

[11] G. L. Acedo and H.-K. Xu, "Iterative methods for strict pseudo-contractions in Hilbert spaces," Nonlinear Analysis, vol. 67, no. 7, pp. 2258-2271, 2007. 


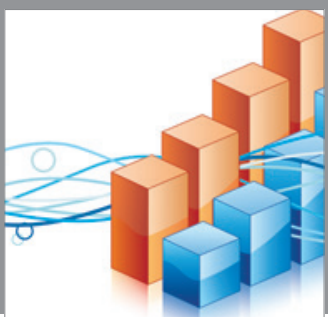

Advances in

Operations Research

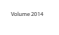

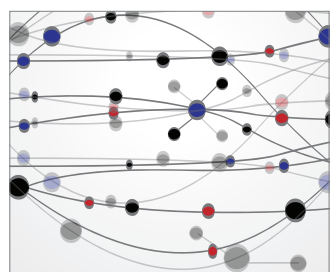

\section{The Scientific} World Journal
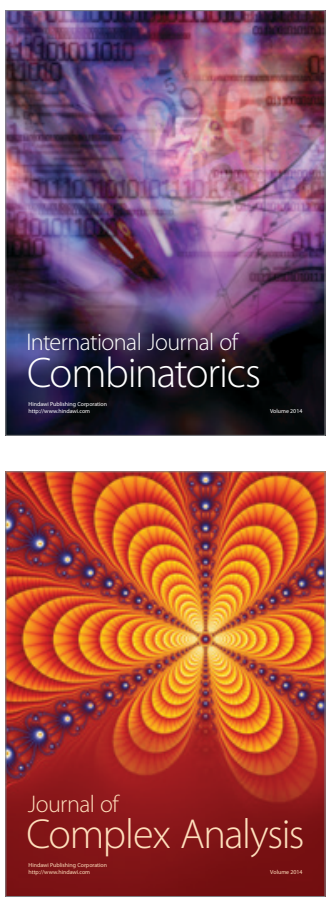

International Journal of

Mathematics and

Mathematical

Sciences
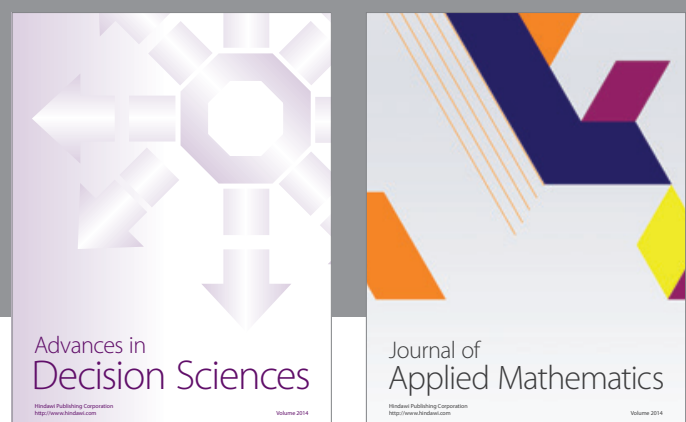

Journal of

Applied Mathematics
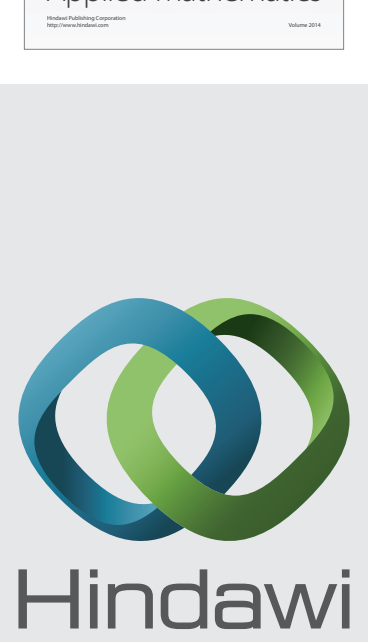

Submit your manuscripts at http://www.hindawi.com
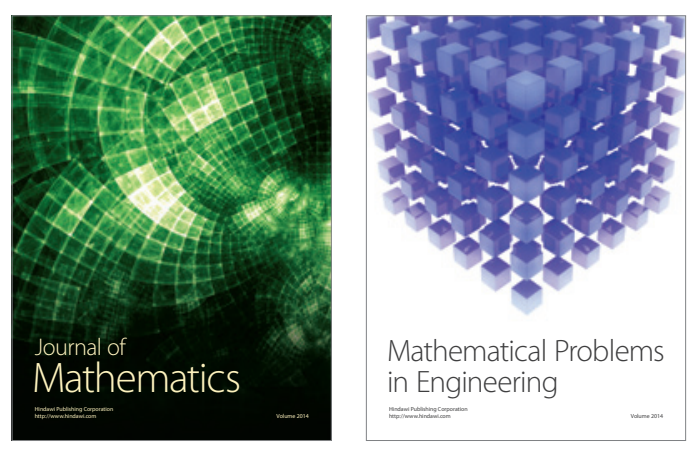

Mathematical Problems in Engineering
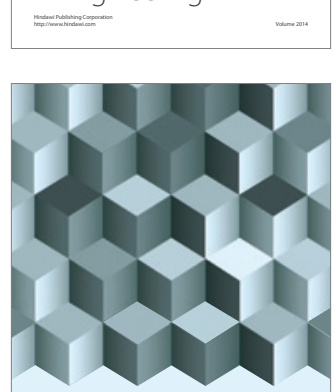

Journal of

Function Spaces
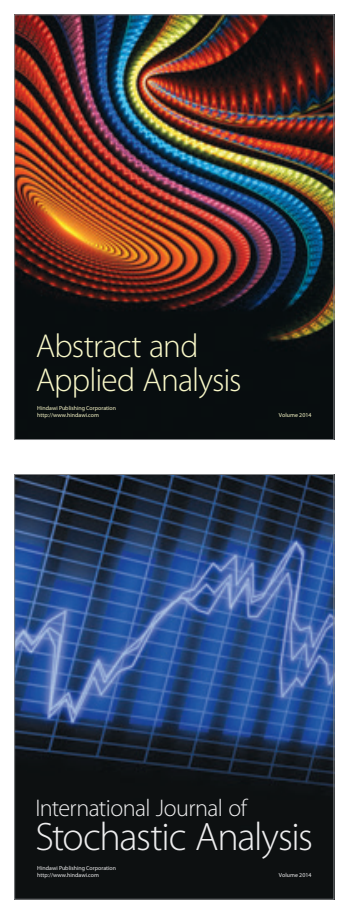

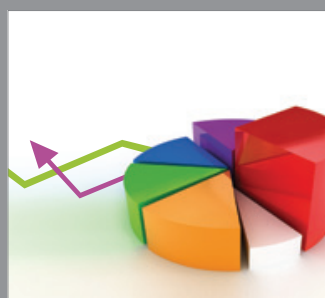

ournal of

Probability and Statistics

Promensencen
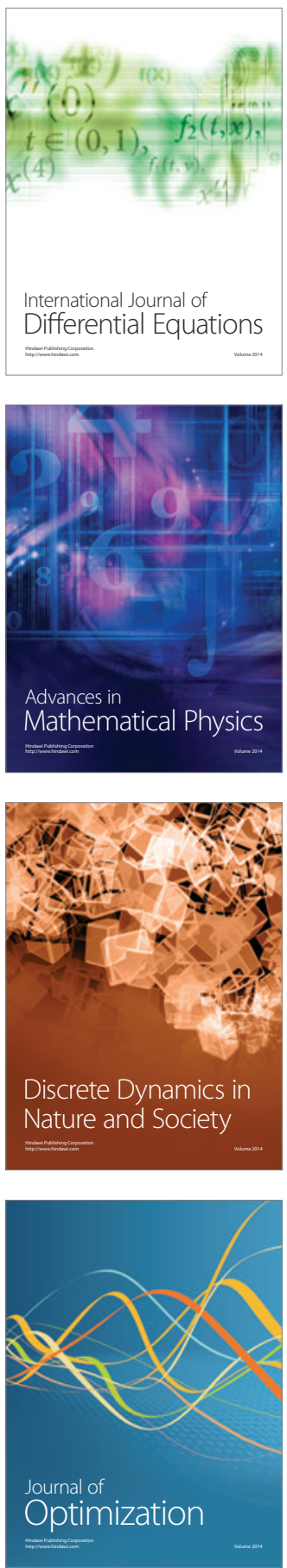